\title{
Reserva Cognitiva e Abertura à Experiência em idosos sem demência: um estudo correlacional
}

\author{
Cognitive reserve and openness to experience in non- \\ demented elderly people: a correlational study \\ Reserva cognitiva y apertura a la experiencia en ancianos \\ sin demencia: un estudio correlacional \\ Joel Jonathan Carvalho Tavares \\ Jayana Ramalho Ventura \\ Bernardino Fernández-Calvo
}

RESUMO: O objetivo desse artigo foi investigar acerca da Reserva Cognitiva (RC) e da Abertura à Experiência (AE) em idosos sem demência. Trata-se de uma pesquisa de campo, descritiva, correlacional e quantitativa, da qual participaram 31 idosos divididos em dois grupos: Saudável e Comprometimento Cognitivo Leve (CCL). Houve correlação moderada, positiva e significativa $(r=0,463 ; p<0,01)$ entre RC e AE. Percebeu-se que os idosos saudáveis $(\mathrm{M}=0,53 ; \mathrm{DP}=0,10)$ apresentaram significativamente $[\mathrm{t}=(29) 3,785 ; \mathrm{p}<0,01]$ maior índice de $\mathrm{RC}$ em comparação aos idosos com CCL $(\mathrm{M}=0,39$; $\mathrm{DP}=0,11)$. Os dados confirmaram que existe associação entre RC e AE, e que idosos saudáveis apresentam maior RC, comparados a idosos com CCL. Este estudo possibilitou conhecer possíveis relações e diferenças subjacentes ao processo de aquisição das reservas cognitivas e de fatores relativos a $\mathrm{AE}$, podendo contribuir para que mais pesquisas sejam feitas, buscando maiores evidências no campo da ciência.

Palavras-chave: Reserva Cognitiva; Abertura à Experiência; Idosos. 
ABSTRACT: This paper aimed to analyze the relationship between Cognitive Reserve $(C R)$ and Openness to Experience $(O E)$ in non-demented elderly people. Descriptive, correlational and quantitative research was undertaken. The sample consisted of thirty-one elderly people divided into two groups: healthy and Mild Cognitive Impairment (MCI). There was a moderate, positive and significant correlation $(r=0.463 ; p<0.01)$ between $C R$ and OE. It was found that the healthy controls $(M=0.53 ; S D=0.10)$ present significantly $[t=(29) 3,785 ; p<0.01]$ higher $C R$ compared to the elderly with MCI $(M=0.39 ; S D=0.11)$. The data confirm that there was an association between $C R$ and $O E$ and that healthy controls have higher $C R$ compared to the MCI group. This study allowed to know possible relationships and differences underlying the acquisition process of cognitive reserves and factors related to $O E$ and may contribute to further research, seeking more evidence in the field of science.

Keywords: Cognitive Reserve; Openness; Elderly.

RESUMEN: El objetivo de este trabajo fui investigar la Reserva Cognitiva (RC) y la Apertura a la Experiencia (AE) en ancianos sin demencia. Es una investigación de campo, descriptiva, correlacional y cuantitativa. Los participantes fueron 31 ancianos divididos en dos grupos: ancianos cognitivamente saludables (GS) y ancianos con deterioro cognitivo leve (DCL). Existió una correlación moderada, positiva y significativa $(r=0.463 ; p<0.01)$ entre $R C$ y AE. El GS $(M=0.53 ; S D=0.10)$ presentó significativamente $[t=(29) 3,785 ; p<0.01]$ mayor índice RC comparado con el DCL $(M=0.39 ; S D=0.11)$. Los datos confirmaron que existió una asociación entre $R C$ y $A E$, y que los ancianos cognitivamente sanos tuvieron una RC más alta en comparación con los ancianos con DCL. Este estudio permitió conocer las posibles relaciones y diferencias subyacentes al proceso de adquisición de reservas cognitivas y factores relacionados con la AE y puede contribuir a una mayor investigación, buscando mayor evidencia en el campo de la ciencia.

Palabras clave: Reserva cognitiva; Apertura a la experiencia; Anciano.

\section{Introdução}

A Reserva Cognitiva $(\mathrm{RC})$ refere-se à capacidade adaptável de processos cognitivos em explicar as diferenças sobre quão suscetíveis são as habilidades cognitivas ou diárias que 
impactam as alterações devidas ao envelhecimento, a patologias ou a lesões cerebrais (Stern, et $a l ., 2018)$. Em outras palavras, RC é a capacidade de resiliência cerebral que as experiências da vida, combinadas a fatores genéticos, influenciam na capacidade de as pessoas lidarem melhor com as doenças cerebrais ou com o processo de envelhecimento (Pettigrew, \& Soldan, 2019).

A Abertura à Experiência (AE) é uma das dimensões do modelo dos Cinco Grandes Fatores da Personalidade, o qual se atribui características relacionadas à flexibilidade de pensamento, abertura para novas experiências, interesses culturais, capacidade de imaginação e fantasia (Hutz, et al., 1998). Mediante estas informações, serão revisadas algumas diferenças entre os aspectos neuropsicológicos, fisiológicos e biológicos do envelhecimento saudável e não saudável.

\section{Aspectos neuropsicológicos do envelhecimento}

O processo de envelhecimento cognitivo é considerado um fenômeno variável e complexo, ou seja, varia de indivíduo para indivíduo o que, muitas vezes, pode dificultar a identificação e a classificação desse fenômeno como um processo normal ou patológico (Apolinário, \& Vernaglia, 2016).

\section{O Processo de Envelhecimento Saudável}

A velhice é uma fase do ciclo de vida do ser humano, podendo ter seu início considerado a partir dos 65 anos de vida, e que é dividida em idosos jovens, com idade entre 65 e 74 anos; idosos velhos, com idade superior a 75 anos; e idosos muito velhos, com mais de 85 anos de idade (Sadock, Sadock, \& Ruiz, 2017). Todavia, no Brasil, de acordo com o art. 2 da lei que dispõe acerca da Política Nacional do Idoso, considera-se idoso o adulto com idade superior a 60 anos (Brasil, 1994).

Os idosos podem ser descritos como saudáveis ou doentes. Para os autores, o processo de envelhecimento se caracteriza através do declínio de forma gradual no funcionamento cardiovascular, respiratório, imunológico, endócrino, e demais sistemas do corpo humano. Todavia, no envelhecimento saudável, permanece a manutenção das capacidades e habilidades físicas e cognitivas, sendo considerado mito a concepção de envelhecimento como uma fase de enfermidades físicas e intelectuais (Sadock, et al., 2017). 
Do ponto de vista neurobiológico ocorre o envelhecimento das células, advindo mudanças na sua estrutura e, consequentemente, no sistema nervoso central, decorrendo também degenerações nos neurônios, sendo mais grave em idosos não saudáveis. Igualmente, aparecem alterações na estrutura e mutações no Ácido Desoxirribonucleico (DNA) e no Ácido Ribonucleico (RNA), sendo afetadas todas as áreas do corpo humano (Sadock, et al., 2017). Além das mudanças estruturais e alterações celulares, ocorrem também a apoptose ${ }^{1}$ e as substituições de células danificadas como estratégia de sobrevivência (Bicalho, \& Cintra, 2013).

Também é possível observar diversas mudanças fisiológicas no processo de senescência dos seres humanos: mudanças na composição corporal, musculatura, modificações ósseas e articulares são visíveis, além do declínio observado nos órgãos dos sentidos como audição, visão, paladar e olfato (Bicalho, \& Cintra, 2013).

Com relação ao Sistema Nervoso (SN), no Sistema Nervoso Autônomo (SNA), as alterações influenciam a capacidade de o idoso enfrentar as mudanças ambientais e a modulação das funções viscerais. Já no que tange ao Sistema Nervoso Central (SNC), as alterações no perfil de neurotransmissores ocorrem de forma menos exacerbadas no envelhecimento saudável, em detrimento do envelhecimento patológico (Ribeiro, \& Cosenza, 2013). Alterações morfológicas e bioquímicas podem ser encontradas com maiores detalhes nos estudos dos referidos autores.

\section{Aspectos patológicos do envelhecimento}

Existem diversos tipos de demências, sendo a doença de Alzheimer a mais comum, seguida de demência vascular e demência com corpos de Levy (Livingston, et al., 2017). A Alzheimer é caracterizada como uma doença neurodegenerativa, a qual envolve um declínio da memória episódica associada a outros déficits cognitivos como os da linguagem, funções executivas, visuoespaciais etc. (Gaugler, James, Johnson, Scholz, \& Weuve, 2016; Souza, \& Teixeira, 2013).

\footnotetext{
${ }^{1}$ Apoptose: uma forma de morte celular programada, o chamado "suicídio celular", sendo diferente de necrose, na qual as células morrem por causa de uma lesão. A apoptose é processo ordenado, no qual o conteúdo da célula é compactado em pequenos pacotes de membrana para a coleta pelas células do sistema imunológico.

Tavares, J. J. C., Ventura, J. R., \& Fernández-Calvo, B. (2019). Reserva Cognitiva e Abertura à Experiência em idosos sem demência: um estudo correlacional. Revista Kairós-Gerontologia, 22(4), 77-97. ISSNprint 1516-2567. ISSNe 2176-901X. São Paulo (SP), Brasil: FACHS/NEPE/PEPGG/PUC-SP
} 
A Demência Frontotemporal é considerada como uma das mais comuns em pessoas com idade abaixo dos 65 anos, e ocorre devido a uma atrofia progressiva nos lóbulos frontais e temporais, acarretando diversas alterações nas funções executivas e outras funções associadas a esses lóbulos (Rosness, Engedal, \& Chemali, 2016). A doença de Parkinson é considerada a segunda causa de desordens neurodegenerativas, cujas características podem incluir: bradicinesia, rigidez, tremor em repouso, marcha lenta e desequilíbrios, constituindo a maior causa de parkinsonismo (Reich, \& Savitt, 2019). As doenças cerebrovasculares são responsáveis pelas demências secundárias às lesões cerebrovasculares. Caracterizadas por demências vasculares, as doenças cerebrovasculares são classificadas em segundo lugar como causa de demências, tendo uma maior incidência relacionada à idade. Reconhecidamente por possuir condições clínicas heterogêneas, as demências vasculares abrangem diversos tipos de demências secundárias a infartos ou hemorragias cerebrais (Souza, \& Teixeira, 2013).

O termo Comprometimento Cognitivo Leve (CCL), ou no original Mild Cognitive Impairment (MCI), foi cunhado por Petersen, et al. (1997), que explica o estado em que uma única função da cognição, frequentemente a memória, fica comprometida em um grau acima da normalidade para a idade, mas que não chega a cumprir critérios para demência. O CCL pode ser considerado como a "fronteira" entre envelhecimento normal e demência, sendo um processo de transição, no qual pessoas que desenvolveram demência, provavelmente desenvolveram CCL em alguma fase anterior à doença (Petersen, et al., 1997; Smith, et al., 1996).

Os critérios diagnósticos para reconhecer um indivíduo idoso com CLL são formados a partir da presença de queixas subjetivas de memória, Atividades de Vida Diária (AVD’s) preservadas, funcionalidade cognitiva geral normal, alterações mnésicas e ausência de demência (Petersen, et al., 1997). Todavia, não é necessário apresentar alterações mnésicas; atualmente o CCL também pode ser classificado em dois subtipos: amnésico e não amnésico, sendo de múltiplos domínios ou único domínio (Malloy-Diniz, et al., 2013). Fatores como pontuações em testes de memórias com desvio-padrão < - 1,5 em relação à média de normalidade populacional para idade e escolaridade, pontuação $\geq 24$ pontos no Mini-Exame do Estado Mental (MEEM) (Folstein, Folstein, \& McHugh, 1975), ou pelo menos 123 pontos no Dementia Rating Scale (DRS) também são considerados critérios diagnósticos para CCL (Canineu, Samara, \& Stella, 2016). 


\section{O construto de reserva cognitiva e suas especificidades}

Para explorar o significado do termo Reserva Cognitiva (RC), torna-se fundamental compreender o que é Reserva e quais as variantes envolvidas nesse construto. Reserva é uma característica estratégica, a que os autores chamam de heurística, que ajuda a explicar as diferenças individuais na cognição, na função, ou na condição clínica de um sujeito, relativo ao processo de envelhecimento e doenças cerebrais. Trata-se de um construto complexo, o qual pode depender de mecanismos cerebrais estruturais e funcionais (Stern, et al., 2018).

Stern (2002) subdividiu a Reserva em dois padrões, o primeiro chama-se modelo passivo de Reserva, o qual inclui a Reserva Cerebral. Neste padrão, o que define a Reserva é a quantidade de dano cerebral que pode ser tolerado antes de atingir o limiar, e surgirem expressões clínicas da patologia. Já no modelo ativo, o qual está incluso a RC, a Reserva é caracterizada pelas diferenças de como a tarefa é processada, sugerindo que o cérebro tenta compensar de forma ativa o dano cerebral.

A reserva cerebral pode ser concebida como um capital neurobiológico - que envolve números de neurônios, sinapses, entre outros - que explicam a variação individual nas características estruturais do cérebro, sendo permissor para algumas pessoas lidarem melhor com o envelhecimento cerebral e patologias de que outras pessoas, antes do surgimento de mudanças clínicas ou cognitivas (Stern, et al., 2018).

Enquanto a Reserva Cerebral pode ser considerada como o "hardware" do modelo passivo de reserva, a RC pode ser definida como o "software" do modelo ativo, o qual consiste na capacidade de o cérebro lidar com as mudanças do envelhecimento ou alterações de uma patologia cerebral, explicado por diferenças individuais de habilidades cognitivas ou do dia a dia, que minimizam ou retardam as manifestações clínicas de uma doença cerebral ou de um processo neurodegenerativo. Este processo pode ser influenciado pela interação de diferenças individuais inatas, determinadas geneticamente, bem como a exposições ao longo da vida (Stern, et al., 2018).

Algumas exposições ao longo da vida são relevantes e podem ser incluídas no rol de diferenças individuais da RC, como: capacidades cognitivas adquiridas no início da vida - por exemplo, inteligência - nível educacional, aspecto ocupacional, atividades físicas e de lazer, bem como engajamento social (Stern, et al., 2018). 
Além das variáveis citadas acima, os desafios intelectuais, como ler e escrever, fazem parte das diferenças individuais que contribuem para a acumulação de reservas ao longo da vida. Até mesmo o status socioeconômico e o nível de envolvimento nos estilos de vida são variáveis descritivas de experiências de vida relevantes para o nível de RC (Pettigrew, \& Soldan, 2019; Sobral, \& Paúl, 2015; Staff, Murray, Deary, \& Whalley, 2004).

A Reserva pode ser fruto de capacidades inatas, somadas aos efeitos das experiências vivenciadas. Entende-se que variáveis como escolaridade, atividades laborais e de lazer são os produtos que formam as reservas, que variam conforme as experiências individuais e as diferenças inatas (Sobral, \& Paúl, 2015).

A RC trata-se de um construto teórico que não pode ser diretamente observado; por isso, tornou-se necessária a construção de instrumentos que possibilitem quantificar e avaliar, de forma empírica, esse conceito (Pettigrew, \& Soldan, 2019).

Sobral e Paúl (2015) apontaram algumas limitações encontradas na produção desses instrumentos, e consideram o conceito de RC bastante complexo, o que levou a proporem diversas propostas para avaliá-lo. A influência de diversas variáveis não pode ser definida por completo, não existindo, assim, consenso formal acerca de quais testes são mais indicados para avaliar a RC. Com isso, os autores chamam a atenção para a dificuldade de explorar variáveis genéticas e ambientais nos instrumentos, mas que, apesar das limitações, existem alguns instrumentos favoráveis para mensuração da $\mathrm{RC}$, citados na obra dos referidos pesquisadores (Sobral, \& Paúl, 2015)

\section{A teoria fatorial da personalidade}

O Modelo dos Cinco Grandes Fatores da Personalidade surgiu nos anos 1980, baseado na teoria do traço. Bastante conhecido na literatura como big five, este novo modelo surgiu a partir de diferentes pesquisas no campo da personalidade (Digman, 1996; McCrae, \& John, 1992).

A primeira das cinco dimensões ou fatores da personalidade propostas na teoria é a extroversão/introversão. Este termo foi cunhado inicialmente por Tupes e Christal (1992) e consiste em características individuais como: discursividade - ou seja, a característica presente em pessoas que gostam de discursar - ser aventureira, ter franqueza, assertividade, sociabilidade etc. (Hutz, et al., 1998). 
Para o segundo fator, é usado o termo em inglês agreeableness, o que, em tradução livre, significa agradabilidade. Este elemento corresponde ao nível de socialização, o qual envolve os traços de altruísmo, maturidade emocional, coperatividade, gentileza, entre outras. Este item também compreende traços negativos como: hostilidade, indiferença, egoísmo e inveja (Tupes, \& Christal, 1992).

O terceiro fator, a conscienciosidade, também conhecido como escrupulosidade ou vontade, engloba as características de personalidade relacionadas à responsabilidade e honestidade, tendo também seu polo investido, compreendendo características contrárias como negligência e irresponsabilidade (Hutz, et al., 1998).

A quarta dimensão da Teoria Fatorial da Personalidade chama-se neuroticismo/estabilidade emocional, fator este que abrange traços que envolvem afeto positivo ou negativo, estabilidade emocional, ansiedade etc. (Hutz, et al., 1998).

O quinto componente é o mais importante para este estudo, pois se trata do traço de intelecto. Também denominado abertura para experiência, esta dimensão envolve características como fantasia e imaginação, flexibilidade de pensamento e abertura para novas experiências e interesses culturais (Hutz, et al., 1998). Este fator será discutido detalhadamente mais adiante.

\section{Definindo Abertura à Experiência}

O fator de AE é capaz de avaliar o quanto uma pessoa é aberta e se interessa por novas situações e experiências. Pessoas abertas possuem uma postura mais criativa e receptiva para diferentes valores, tentam coisas novas e vão a novos lugares, sendo também inerentemente curiosas, criativas, imaginativas, possuem uma grande necessidade de novidades e variedades, procurando sempre ter tais experiências. Apesar de pertencer ao fator do intelecto, a abertura não está relacionada diretamente com a inteligência. Esta dimensão possui maior conexão com os comportamentos exploratórios citados anteriormente (McCrae, \& Sutin, 2009; Nunes, \& Hutz, 2007; Sutin, 2017).

O oposto de pessoas abertas são aqueles indivíduos fechados, que preferem o convencional, são mais conservadores, dogmáticos e possuem menor flexibilidade em suas crenças (Sutin, 2017). 
Estudos mostram que pessoas inseridas em um sistema de crenças com concepções dogmáticas e fundamentalistas possuem um menor índice de AE (Saroglou, 2002; Saroglou, \& Muñoz-García, 2008). Já no que tange a visões políticas, pessoas menos abertas têm uma maior tendência a se identificarem com posturas etnocêntricas e doutrinas autoritárias (Butler, 2000). Fica claro que existem diferenças nas atitudes de pessoas com diferentes traços de personalidade; tais atitudes podem influenciar diversos outros fatores presentes na vida de un sujeito.

Fatores como inteligência, hábitos saudáveis de vida e de trabalho etc. podem ser capazes de compensar as deficiências, que são progressivas no envelhecimento. A personalidade é um dos fatores que influenciam a cognição e pode ser crucial em determinar o risco para declínio cognitivo em idosos (Canineu, et al., 2016).

Perante o que foi colocado acerca da dimensão de AE e o construto de RC abordado anteriormente, pode-se inferir que características comuns às pessoas abertas à experiência são similares a algumas variáveis mensuráveis no construto de RC, tornando-se, portanto, relevante um estudo correlacional entre RC e AE.

O objetivo deste artigo é investigar acerca da RC e AE em idosos sem demência, com o propósito de descobrir se há correlação entre os índices nesses dois construtos, bem como comparar tais índices em idosos com CCL e idosos com envelhecimento saudável, verificando possíveis diferenças de idade. Esta pesquisa poderá permitir o levantamento de novas hipóteses, e, consequentemente, novos estudos com o intuito de aprofundar o conhecimento de fatores que podem influenciar o processo de envelhecimento.

\section{Método}

Trata-se de uma pesquisa de campo, descritiva, de delineamento correlacional e de natureza quantitativa. Os dados foram coletados nas dependências do Serviço de Neuropsicologia do Envelhecimento (SENE), localizado na Clínica-Escola de Psicologia da Universidade Federal da Paraíba (UFPB). A técnica de escolha amostral foi a não probabilística por conveniência, na qual participaram 31 idosos advindos do SENE, no ano de 2019, divididos em dois grupos: Grupo Saudável (GS) e Grupo CCL (GC).

Para ambos os grupos, os critérios de elegibilidade foram: 
- Critérios de inclusão: idosos com 60 a 84 anos; alfabetizados; de ambos os sexos; de naturalidade brasileira; de todas as classes econômicas e sociais, raças e etnias, e que aceitaram a participar do estudo e assinaram o Termo de Consentimento Livre e Esclarecido (TCLE);

- Critérios de exclusão: possuir diagnóstico de qualquer tipo de demência; presença de déficit auditivo-visual não corrigido que impossibilite a aplicação dos instrumentos e alterações neurológicas diagnosticadas.

Para o GC, os critérios de elegibilidade específicos foram:

- Critérios de inclusão: queixas subjetivas de memórias ou queixas cognitivas em outros domínios; atividades de vida diária sem nenhuma ou mínima perda de funcionalidade, de acordo com os critérios de Petersen, et al. (1997; Petersen, \& Morris, 2005), para CCL e/ou declínio cognitivo comparado à idade e a escolaridade, calculados a partir do resultado no MEEM com $\mathrm{Z}>-1,5$.

- Critérios de exclusão: impressões clínicas compatíveis com demência segundo os critérios do DSM-V para Transtornos Neurocognitivos (American Psychiatric Association, 2014) e/ou escore Z, calculado a partir do resultado do MEEM com Z > - 2,0 para a idade e escolaridade, de acordo com os estudos normativos de Brucki, Nitrini, Caramelli, Bertolucci, \& Okamoto (2003).

Para o GS, os critérios de elegibilidade específicos foram:

- Critérios de inclusão: função cognitiva geral preservada e/ou pontuação no MEEM calculada a partir do escore $Z$, obtendo-se resultado $Z<-1,5$, esperada para a escolaridade e a idade do sujeito, comparados aos dados normativos do instrumento, segundo Brucki, et al. (2003).

- Critérios de exclusão: relato de perda funcional significativa das atividades básicas e instrumentais de vida diária, histórico de abuso de álcool ou outras drogas, presença de transtornos psiquiátricos diagnosticados e/ou escore $\mathrm{Z}$ calculado a partir do MEEM com resultado $Z>-1,5$ para a idade e escolaridade, de acordo com os estudos de normatização de Brucki, et al. (2003).

Para a coleta de dados, foram utilizados cinco instrumentos:

- Questionário sociodemográfico, que teve como objetivo coletar informações sobre gênero, idade, nacionalidade, estado civil, escolaridade e renda financeira;

- Questionário de histórico clínico, que teve o intuito de coletar informações acerca das características clínicas do participante, a partir da coleta dos dados presentes no prontuário. 
Este questionário assinalou a presença ou ausência de queixas subjetivas de memória, déficits auditivos ou visuais, perda funcional significativa das atividades básicas e instrumentais de vida diária, história de abusos de álcool ou outras drogas, alterações neurológicas e transtornos psiquiátricos diagnosticados, a fim de atender aos critérios de elegibilidade da pesquisa;

- Escala Fatorial de Abertura à Experiência (EFA), proposta por Vasconcellos (2007) e Vasconcellos, \& Hutz (2008), contendo 42 itens distribuídos em 3 fatores: atitudes, hábitos e valores e fantasia, com respostas em uma escala tipo likert, variando de 1 a 7 pontos;

- Questionário de Reserva Cognitiva (QRC), modificado de Rami González, et al. (2011), contendo 15 itens distribuídos em 3 fatores: atividades cognitivas, atividades sociais e atividades físicas;

- Mini-Exame do Estado Mental (MEEM) em sua versão brasileira normatizada por Brucki, et al. (2003), composta por 11 itens subdivididos em 20 subitens. Trata-se de um teste de rastreio cognitivo, o qual avalia diversos domínios; foi utilizado nesta pesquisa como instrumento de triagem para os grupos amostrais, baseados nos critérios de elegibilidade deste estudo.

Os dados coletados por meio de todos os instrumentos foram processados pelo programa estatístico SPSS versão 22.0.0.0, utilizando-se estatística descritiva e inferencial. Com base nos dados coletados, foram realizadas análises estatísticas de frequência, correlação e comparação de médias, a fim de responder aos objetivos deste estudo. Para analisar possíveis associações entre RC e AE, foi utilizado o coeficiente de correlação R de Pearson e, para comparar as médias dos índices de RC e AE, foi utilizado o teste paramétrico T de Student.

Este estudo foi realizado, considerando-se os aspectos éticos, referentes à pesquisa com seres humanos, consoante com a Resolução 466/12, submetido ao Comitê de Ética em Pesquisa do Centro Universitário de João Pessoa (UNIPÊ) e aprovado sob o parecer CAEE 13419319.6.0000.5176.

\section{Resultados}

Participaram 31 idosos com idade entre 60 e 84 anos $(M=66,97$; $D P=5,84)$, compostos em sua maioria por idosos jovens $(87,1 \%)$, do sexo feminino $(74,2 \%)$, casados $(51,6 \%)$, com 
ensino superior $(71,0 \% ; \mathrm{M}=14,61 ; \mathrm{DP}=4,01)$ e renda acima de 3 salários mínimos $(61,3 \%)$ (Tabela 1).

Através do teste de rastreio MEEM e do questionário de histórico clínico, foram definidos os participantes do GS e GC. Para o GS, composto de idosos saudáveis, foram selecionados 19 idosos (61,3\%); para o GC, formado por idosos com impressão clínica compatível com CCL, foram selecionados 12 indivíduos $(38,7 \%)$, com relação ao total de participantes; nenhum indivíduo (0\%) foi excluído dos grupos (Tabela 1).

Tabela 1 - Perfil sociodemográfico dos participantes da pesquisa $(n=31)$

\begin{tabular}{|c|c|c|c|c|c|c|}
\hline & \multicolumn{2}{|c|}{ TOTAL $(\mathbf{n}=31)$} & \multicolumn{2}{|c|}{ GS $(n=19)$} & \multicolumn{2}{|c|}{ GC $(n=12)$} \\
\hline & $\mathrm{f}$ & $\%$ & $\mathrm{f}$ & $\%$ & $\mathrm{f}$ & $\%$ \\
\hline \multicolumn{7}{|l|}{ Sexo } \\
\hline Masculino & 08 & $25,8 \%$ & 05 & $62,5 \%$ & 03 & $37,5 \%$ \\
\hline Feminino & 23 & $74,2 \%$ & 14 & $60,9 \%$ & 09 & $39,1 \%$ \\
\hline \multicolumn{7}{|l|}{ Faixa Etária } \\
\hline Idosos Jovens (60 a 74 anos) & 27 & $87,1 \%$ & 15 & $55,6 \%$ & 12 & $44,4 \%$ \\
\hline Idosos Velhos ( $\geq 75$ anos) & 04 & $12,9 \%$ & 04 & $100 \%$ & 00 & $0 \%$ \\
\hline \multicolumn{7}{|l|}{ Estado Civil } \\
\hline Solteiro & 08 & $25,8 \%$ & 05 & $62,5 \%$ & 03 & $37,5 \%$ \\
\hline Casado & 16 & $51,6 \%$ & 08 & $50,0 \%$ & 08 & $50,0 \%$ \\
\hline Divorciado & 03 & $9,7 \%$ & 02 & $66,7 \%$ & 01 & $33,3 \%$ \\
\hline Viúvo & 04 & $12,9 \%$ & 04 & $100 \%$ & 00 & $0 \%$ \\
\hline \multicolumn{7}{|l|}{ Renda financeira } \\
\hline Até 1 salário & 04 & $12,9 \%$ & 02 & $50,0 \%$ & 02 & $50,0 \%$ \\
\hline Entre 1 e 3 salários & 08 & $25,8 \%$ & 03 & $37,5 \%$ & 05 & $62,5 \%$ \\
\hline Entre 4 e 5 salários & 10 & $32,3 \%$ & 06 & $60,0 \%$ & 04 & $40,0 \%$ \\
\hline Acima de 5 salários & 09 & $29,0 \%$ & 08 & $88,9 \%$ & 01 & $11,1 \%$ \\
\hline \multicolumn{7}{|l|}{ Escolaridade (em anos de estudo) } \\
\hline 1 a 4 anos (ensino básico) & 01 & $3,2 \%$ & 00 & $0 \%$ & 01 & $100 \%$ \\
\hline 5 a 8 anos (ensino fundamental) & 01 & $3,2 \%$ & 01 & $100 \%$ & 00 & $0 \%$ \\
\hline 9 a 11 anos (ensino médio) & 07 & $22,6 \%$ & 03 & $42,9 \%$ & 04 & $57,1 \%$ \\
\hline$\geq 12$ anos (ensino superior/pós-graduação) & 22 & $71,0 \%$ & 15 & $68,2 \%$ & 07 & $31,8 \%$ \\
\hline
\end{tabular}

Fonte: Dados coletados da amostra obtida.

Legenda: $\mathrm{f}$ = frequência; $\mathrm{GS}$ = grupo saudável; $\mathrm{GC}=$ grupo CCL.

Com base na análise estatística inferencial, observa-se que há uma correlação moderada, positiva e significativa $(\mathrm{r}=0,463 ; \mathrm{p}<0,01)$ entre $\mathrm{RC}$ e $\mathrm{AE}$. Isso significa que, à medida que os níveis de RC aumentam, os níveis de AE aumentam. Também houve uma correlação positiva, moderada e significativa entre AE e o fator atividades cognitivas $(r=0,451 ; p<0,05)$; correlação positiva, fraca mas significativa, entre o fator atitude e RC $(\mathrm{r}=0,369 ; \mathrm{p}<0,05)$; correlação positiva, moderada e significativa $(r=0,412 ; \mathrm{p}<0,05)$ entre o fator atitude $\mathrm{e}$ 
atividades cognitivas; assim como correlação moderada, positiva e significativa $(r=0,430 ; p<$ $0,05)$ entre RC e o fator hábitos e valores (Tabela 2).

Tabela 2 - Coeficientes de correlação de Pearson entre RC e AE incluindo os fatores

\begin{tabular}{ccccc}
\hline & AE & Atitude & Hábitos e valores & Fantasia \\
\hline RC & $0,463^{* *}$ & $0,369^{*}$ & $0,430^{*}$ & 0,035 \\
Atividades cognitivas & $0,451^{*}$ & $0,412^{*}$ & 0,343 & 0,112 \\
Atividades físicas & 0,079 & $-0,036$ & 0,242 & $-0,188$ \\
Atividades sociais & 0,352 & 0,255 & 0,344 & 0,031 \\
\hline
\end{tabular}

Fonte: Dados coletados da amostra obtida.

Legenda: $\mathrm{RC}=$ Reserva Cognitiva; $\mathrm{AE}=$ Abertura à Experiência .

$* \mathrm{P}<0,05 ; * * \mathrm{P}<0,01$.

Com relação aos idosos que foram divididos nos grupos GS (idosos saudáveis) e GC (idosos com CCL), foi verificado que houve diferença nos índices de RC e AE entre os idosos de ambos os grupos.

No que se refere à faixa etária dos idosos, foram comparados os índices de $\mathrm{RC}$ e $\mathrm{AE}$ entre idosos jovens (entre 60 e 74 anos) e idosos velhos ( $\geq 75$ anos).

De acordo com os resultados obtidos para os GS e GC, percebe-se que os idosos saudáveis $(\mathrm{M}=0,53 ; \mathrm{DP}=0,10)$ apresentaram significativamente $[\mathrm{t}=(29) 3,785 ; \mathrm{p}<0,01]$ maior índice de RC, em comparação aos idosos com CCL (M =0,39; DP =0,11).

É possível observar também diferenças significativas [t $=(29) 3,067 ; p<0,01]$ no fator atividades cognitivas, indicando que os idosos saudáveis $(\mathrm{M}=0,56 ; \mathrm{DP}=0,12)$, obtiveram maior desempenho de atividades cognitivas do que idosos com $\operatorname{CCL}(\mathrm{M}=0,41 ; \mathrm{DP}=0,15)$.

No fator atividades físicas $[\mathrm{t}=(28,34) 2,388 ; \mathrm{p}<0,05]$, os idosos saudáveis $(\mathrm{M}=0,55$; $\mathrm{DP}=0,26)$ apresentaram maior média do que os idosos com impressão clínica compatível com CCL $(\mathrm{M}=0,35 ; \mathrm{DP}=0,14)$, comparados especificamente nesse fator do instrumento $\mathrm{QRC}$ (Tabela 3). 
Tabela 3 - Comparações das médias de RC e AE entre os grupos de estudo

\begin{tabular}{ccccccc}
\hline & \multicolumn{3}{c}{ GRUPOS COGNIÇÃO } & \multicolumn{3}{c}{ GRUPOS ETÁRIO } \\
\cline { 2 - 7 } $\mathbf{N}$ & SAUDÁVEL & CCL & TESTE & JOVENS & VELHOS & TESTE \\
& 19 & 12 & 31 & 27 & 4 & 31 \\
RC & $\mathrm{M} \pm \mathrm{DP}$ & $\mathrm{M} \pm \mathrm{DP}$ & $\mathrm{T}$ & $\mathrm{M} \pm \mathrm{DP}$ & $\mathrm{M} \pm \mathrm{DP}$ & $\mathrm{T}$ \\
Ativ. cognitivas & $0,53 \pm 0,10$ & $0,39 \pm 0,11$ & $3,785^{* *}$ & $0,47 \pm 0,13$ & $0,49 \pm 0,06$ & $-0,285$ \\
Ativ. físicas & $0,55 \pm 0,12$ & $0,41 \pm 0,15$ & $3,067^{* *}$ & $0,50 \pm 0,16$ & $0,51 \pm 0,13$ & $-0,121$ \\
Ativ. sociais & $0,45 \pm 0,14$ & $0,35 \pm 0,14$ & $2,388^{*}$ & $0,47 \pm 0,23$ & $0,53 \pm 0,33$ & $-0,484$ \\
AE & $0,59 \pm 0,09$ & $0,58 \pm 0,14$ & 1,678 & $0,41 \pm 0,15$ & $0,43 \pm 0,13$ & $-0,166$ \\
Atitude & $0,79 \pm 0,09$ & $0,80 \pm 0,14$ & $-0,337$ & $0,60 \pm 0,09$ & $0,52 \pm 0,07$ & 1,647 \\
hábitos e valores & $0,48 \pm 0,15$ & $0,41 \pm 0,12$ & 1,266 & $0,81 \pm 0,10$ & $0,71 \pm 0,13$ & 1,643 \\
Fantasia & $0,45 \pm 0,14$ & $0,52 \pm 0,13$ & $-1,257$ & $0,48 \pm 0,14$ & $0,37 \pm 0,15$ & 1,320 \\
\hline
\end{tabular}

Fonte: Dados coletados da amostra pesquisada.

Legenda: $\mathrm{N}=$ Número amostral; $\mathrm{M}=$ Média; \pm DP = Desvio-padrão; $\mathrm{T}$ = Teste-T de Student.

$* \mathrm{P}<0,05 ; * * \mathrm{P}<0,01$.

Quando comparados em relação ao nível de $\mathrm{AE}$, verifica-se que não há diferença estatisticamente significativa $[t=(29) 0,337 ; p>0,05]$ entre idosos saudáveis $(\mathrm{M}=0,59$; $\mathrm{DP}=$ $0,09)$ e idosos com CCL $(\mathrm{M}=0,58 ; \mathrm{DP}=0,10)$ (Tabela 3).

Para os grupos etários, os resultados mostram que os idosos jovens $(M=0,47 ; \mathrm{DP}=$ $0,13)$ não evidenciaram diferença significativa $[t=(29)-0,285 ; \mathrm{p}>0,05]$ em relação aos idosos velhos $(M=0,49 ; \mathrm{DP}=0,06)$ nas médias de $\mathrm{RC}$, bem como em todos os fatores analisados individualmente.

Similarmente, não houve diferença significativa nas médias de $A E[t=(29) 1,647 ; p>$ $0,05]$ entre os idosos jovens $(M=0,60 ; \mathrm{DP}=0,09)$ e os idosos velhos $(\mathrm{M}=0,52 ; \mathrm{DP}=0,07)$, resultado esse que se confirma também em todos os fatores de $\mathrm{AE}$ analisados particularmente (Tabela 3).

\section{Discussão}

A partir dos objetivos definidos para este estudo, é possível observar, através dos resultados da pesquisa, que se confirma a existência de correlação positiva entre os construtos de RC e AE.

A fundamentação teórica de ambos os construtos aponta algumas similaridades que podem ser observadas, por exemplo, Stern, et al. (2018) colocam que diferenças individuais e exposições ao longo da vida podem ser relevantes para a formação da RC. 
Fatores como: nível de escolaridade, ocupações, engajamento social, atividades de lazer, entre outras, são aspectos formadores de RC e, dessa forma, se assemelham aos aspectos que mensuram um maior nível de AE. Para Nunes e Hutz (2007), pessoas com altos índices de AE são mais curiosas e abertas para novas experiências.

Desse modo, pode-se inferir que pessoas com maior abertura para novas experiências podem também pontuar maiores níveis de RC, pois, por serem mais abertas a novas vivências, podem tender a se envolver em atividades que aumentem as reservas cognitivas como, por exemplo: aprender uma nova língua, tocar um instrumento etc. Para Pettigrew e Soldan (2019), os fatores socioeconômicos e o nível de envolvimento nos estilos de vida são elementos de experiências de vida importantes para os índices de RC.

Com base nos resultados que verificam possíveis diferenças nas médias de RC entre os GS e GC, é possível compreender que os idosos saudáveis possuem maior RC do que os idosos com CCL. Resultado que pode corroborar os achados de Pettigrew e Soldan (2019), que afirmam que maiores índices de RC estão associados a menor risco de desenvolver CCL ou demência. Para os autores, o efeito protetor da RC pode conferir-se como uma ferramenta de preservação da função cognitiva e bem-estar na velhice.

Sattler, Toro, Schönknecht e Schröder (2012), em um estudo longitudinal, apontam que sujeitos cognitivamente ativos, com nível educacional elevado (ensino superior) e alto status socioeconômico, têm menores riscos de desenvolver CCL e doença de Alzheimer. Para os autores, o alto nível de atividades cognitivas de lazer, nesses sujeitos, opera como fator protetivo contra CCL e Alzheimer, por meio do aumento de RC.

No que tange aos resultados que investigam possíveis diferenças quanto aos índices de $\mathrm{AE}$ em idosos saudáveis e com CCL, é possível verificar que não se confirmam diferenças significativas nas médias desses construtos, sugerindo-se que os idosos saudáveis possuem semelhante índice de AE comparados aos idosos com CCL. Todavia, vale ressaltar que são necessários mais estudos, a fim de confirmar ou rejeitar estes dados.

Apesar da escassez de estudos acerca das variáveis citadas, há estudos nos quais se sugere que idosos com maior abertura, apresentam melhor desempenho nos testes de memória imediata e tardia, bem como maior habilidade nas atividades funcionais de vida diária. Os autores também fazem ver que a imaginação ativa dos sujeitos mais abertos à experiência pode contribuir para que os mesmos mantenham a memória em completo funcionamento até os estágios mais avançados da vida (Gregory, Nettelbeck, \& Wilson, 2010). 
Quanto aos resultados que avaliam as diferenças de RC e AE por faixa etária, é possível inferir que não foram confirmadas as hipóteses de que os idosos jovens apresentam maior RC e AE, quando comparados com idosos velhos.

Já em relação ao construto de AE, apesar de os idosos jovens apresentarem maior média de abertura do que idosos velhos, tais dados não são estatisticamente significativos, o que permite levantar algumas hipóteses acerca de fatores que podem interferir nos resultados como: baixo número amostral de idosos velhos, ausência de idosos velhos com CCL na amostra pesquisada, entre outros fatores. Existem poucos estudos disponíveis que visam a investigar especificamente essas variáveis; ainda é possível encontrar estudos que abordam as diferenças de idade entre adultos de meia-idade e adultos idosos, como o estudo transversal de Donnellan e Lucas (2008), o qual aponta para existência de associação negativa entre AE e faixa etária.

\section{Conclusão}

Diante dos dados coletados, e do referencial teórico estudado, é possível depreender que o construto de RC está sob a influência de diversos fatores, podendo a personalidade ser um dos aspectos importantes para a aquisição de reservas cognitivas. No que se refere aos aspectos de faixa etária e o estado de saúde cognitiva do idoso, sabe-se que essas condições devem ser consideradas no estudo do processo de envelhecimento patológico e saudável, pois as possíveis associações com esses elementos podem possibilitar compreender melhor os aspectos subjacentes aos padrões de normalidade do processo saúde-doença no envelhecimento.

Os dados confirmam que existe associação entre RC e AE e que os idosos saudáveis apresentam maior RC, comparados a idosos com CCL. Quando avaliadas as diferenças na média de $\mathrm{AE}$ entre os grupos de idosos saudáveis e com CCL, não são observáveis diferenças significativas, bem como em relação aos índices de ambos os construtos nos grupos de idosos por faixa etária. Todavia, é necessário o desenvolvimento de outros estudos, a fim de aprofundar e complementar os achados sobre este tema, buscando evidenciar a validade dos resultados analisados nesta pesquisa.

Diante das limitações do presente estudo, não se pode deixar de citar que condições como: baixo número amostral, dificuldade de estabelecimento de sujeitos no grupo saudável e CCL; baixa representatividade de sujeitos do sexo masculino, pessoas com escolaridade abaixo 
de 12 anos de estudos, idosos velhos saudáveis e com CCL; entre outros elementos, são fatores que podem limitar os resultados encontrados nesta pesquisa.

Além disso, vale ressaltar a necessidade de se efetuarem levantamentos acerca desse tema com idosos muito velhos ( $\geq 85$ anos), grupo que não foi explorado neste estudo devido à carência de indivíduos com esse perfil dispostos a participarem da pesquisa e a dificuldade de instrumentos validados para esse segmento populacional.

Este estudo possibilitou conhecer possíveis relações e diferenças subjacentes ao processo de aquisição das reservas cognitivas e de fatores relativos a $\mathrm{AE}$. Conhecer condições que possam estar ligadas a aspectos importantes para a manutenção da saúde cognitiva é uma importante busca a ser empreendida; a análise realizada pode contribuir para que mais pesquisas sejam feitas com o intuito de verificar essas condições em diferentes circunstâncias, buscando maiores evidências no campo da ciência.

Portanto, diante dos resultados obtidos e dos conteúdos teóricos discutidos, pode-se concluir que estudar a personalidade e a cognição é um desafio para o pesquisador do campo da gerontologia; é através do levantamento de novos estudos que se torna possível desenvolver ações que podem trazer mais longevidade e qualidade de vida para o idoso.

\section{Referências}

American Psychiatric Association. (2014). Manual diagnóstico e estatístico de transtornos mentais. ( $5^{\mathrm{a}}$ ed.). Porto Alegre, RS: Artmed.

Apolinário, D., \& Vernaglia, I. F. G. (2016). Estilo de Vida Ativo e Cognição na Velhice. In: E. V. Freitas, \& L. Py (Eds.). Tratado de Geriatria e Gerontologia (4ª ed., Cap. 136, 32353246). Rio de Janeiro, RJ: Guanabara Koogan.

Bicalho, M. A. C., \& Cintra, M. T. G. (2013). Modificações fisiológicas sistêmicas no envelhecimento. In: L. F. Malloy-Diniz, D. Fuentes, \& R. M. Cosenza. (Orgs.). Neuropsicologia do Envelhecimento: Uma abordagem multidimensional (Cap. 2, 43-63). Porto Alegre, RS: Artmed.

Brasil. Lei $n .^{\circ}$ 8.842, de 04 de janeiro de 1994. (1994, 04 de janeiro). Dispõe sobre a Política Nacional do Idoso, cria o Conselho Nacional do Idoso e dá outras providências.

Brucki, S. M. D., Nitrini, R., Caramelli, P., Bertolucci, P. H. F., \& Okamoto, I. H. (2003). Sugestões para o uso do mini-exame do estado mental no Brasil. Arquivos de NeuroPsiquiatria, 61(3B), 777-781. Recuperado em 01 dezembro, 2018, de: https://doi.org/10.1590/S0004-282X2003000500014. 
Butler, J. C. (2000). Personality and emotional correlates of right-wing authoritarianism. Social Behavior and Personality: an International Journal, 28(1), 1-14. Recuperado em 01 dezembro, 2018, de: https://doi.org/10.2224/sbp.2000.28.1.1.

Canineu, P. R., Samara, A. B., \& Stella, F. (2016). Transtorno Neurocognitivo Leve. In E. V. Freitas \& L. PY (Eds.), Tratado de Geriatria e Gerontologia (4ª ed., Cap. 21, 585-603). Rio de Janeiro, RJ: Guanabara Koogan.

Digman, J. M. (1996). The curious history of the five-factor model. In J. S. Wiggins (Ed.), The Five-Factor Model of Personality: Theoretical Perspectives (Chap. 1, 1-20). New York, USA: Guilford Press.

Donnellan, M. B., \& Lucas, R. E. (2008). Age differences in the big five across the life span: Evidence from two national samples. Psychology and Aging, 23(3), 558-566. Recuperado em 01 dezembro, 2018, de: https://doi.org/10.1037/a0012897.

Folstein, M. F., Folstein, S. E., \& McHugh, P. R. (1975). "Mini-mental state": A pratical method for grading the cognitive state of patients for the clinician. Journal of Psychiatric Research, 12(3), 189-198. Recuperado em 01 dezembro, 2018, de: https://doi.org/10.1016/0022-3956(75)90026-6.

Gaugler, J., James, B., Johnson, T., Scholz, K., \& Weuve, J. (2016). 2016 Alzheimer's disease facts and figures. Alzheimer's and Dementia, 12(4), 459-509. Recuperado em 01 dezembro, 2018, de: https://doi.org/10.1016/j.jalz.2016.03.001.

Gregory, T., Nettelbeck, T., \& Wilson, C. (2010). Openness to experience, intelligence, and successful ageing. Personality and Individual Differences, 48(8), 895-899. Recuperado em 01 dezembro, 2018, de: https://doi.org/10.1016/j.paid.2010.02.017.

Hutz, C., Nunes, C., Silveira, A., Serra, J., Anton, M., \& Wieczorek, L. (1998). O desenvolvimento de marcadores para a avaliação da personalidade no modelo dos cinco grandes fatores. Psicologia: Reflexão e Crítica, 11(2), 395-411. Recuperado em 01 dezembro, 2018, de: https://doi.org/10.1590/S0102-79721998000200015.

Livingston, G., Sommerlad, A., Orgeta, V., Costafreda, S. G., Huntley, J., Ames, D., ... Mukadam, N. (2017). Dementia prevention, intervention, and care. The Lancet, 390(10113), 2673-2734. Recuperado em 01 dezembro, 2018, de: https://doi.org/10.1016/S01406736(17)31363-6.

Malloy-Diniz, L. F., Abreu, N., Bertola, L., Fuentes, D., Antunes, A. M., Paula, J. J., \& Haase, V. G. (2013). O exame neuropsicológico do idoso. In: L. F. Malloy-Diniz, D. Fuentes, \& R. M. Cosenza. (Orgs.), Neuropsicologia do Envelhecimento: Uma abordagem multidimensional (pp. 243-264). Porto Alegre, RS: Artmed.

McCrae, R. R., \& John, O. P. (1992). An Introduction to the Five-Factor Model and Its Applications. Journal of Personality, 60(2), 175-215. Recuperado em 01 dezembro, 2018, de: https://doi.org/10.1111/j.1467-6494.1992.tb00970.x.

McCrae, R., \& Sutin, A. (2009). openness to experience. In: M. . R. Leary, \& H. R. Hoyle, (Eds.). Handbook of Individual Differences in Social Behavior (Chap. 17, 257-273). New York: The Guilford Press. 
Nunes, C. H. S. da S., \& Hutz, C. S. (2007). Construção e validação da escala fatorial de Socialização no modelo dos Cinco Grandes Fatores de Personalidade. Psicologia: Reflexão e Crítica, 20(1), 20-25. Recuperado em 01 dezembro, 2018, de: https://doi.org/10.1590/S010279722007000100004.

Petersen, R. C., \& Morris, J. C. (2005). Mild Cognitive Impairment as a Clinical Entity and Treatment Target. Archives of Neurology, 62(7), 1160-1163. Recuperado em 01 dezembro, 2018, de: https://doi.org/10.1001/archneur.62.7.1160.

Petersen, R. C., Smith, G. E., Waring, S. C., Ivnik, R. J., Kokmen, E., \& Tangelos, E. G. (1997). Aging, Memory, and Mild Cognitive Impairment. International Psychogeriatrics, 9(S1), 6569. Recuperado em 01 dezembro, 2018, de: https://doi.org/10.1017/S1041610297004717.

Pettigrew, C., \& Soldan, A. (2019). Defining Cognitive Reserve and Implications for Cognitive Aging. Current Neurology and Neuroscience Reports, 19(1), 1-12. Recuperado em 01 dezembro, 2018, de: https://doi.org/10.1007/s11910-019-0917-z.

Rami González, L., Valls Pedret, C., Bartrés Faz, D., Caprile Elola-Olaso, C., Solé Padullés, C., Castellví Sampol, M., ... Molinuevo Guix, J. L. (2011). Cuestionario de reserva cognitiva. Valores obtenidos en población anciana sana y con enfermedad de Alzheimer. Revista de Neurología, 52(04), 195-201. Recuperado em 01 dezembro, 2018, de: https://doi.org/10.33588/rn.5204.2010478.

Reich, S. G., \& Savitt, J. M. (2019). Parkinson's Disease. Medical Clinics of North America, 103(2), 337-350. Recuperado em 01 dezembro, 2018, de: https://doi.org/10.1016/j.mcna.2018.10.014.

Ribeiro, A. M., \& Cosenza, R. M. (2013). Envelhecimento normal do sistema nervoso. In L. F. Mallot-Diniz, \& R. M. Cosenza. (Orgs.). Neuropsicologia do Envelhecimento: Uma abordagem multidimensional (Cap. 4, 78-99). Porto Alegre, RS: Artmed..

Rosness, T. A., Engedal, K., \& Chemali, Z. (2016). Frontotemporal Dementia: An Updated Clinician's Guide. Journal of Geriatric Psychiatry and Neurology, 29(5), 271-280. Recuperado em 01 dezembro, 2018, de: https://doi.org/10.1177/0891988716654986.

Sadock, B. J., Sadock, V. A., \& Ruiz, P. (2017). Psiquiatria geriátrica. In Compêndio de Psiquiatria: ciência do comportamento e psiquiatria clínica $\left(11^{\mathrm{a}}\right.$ ed, Cap. 33, 1334-1351). Porto Alegre, RS: Artmed.

Saroglou, V. (2002). Religion and the five factors of personality: a meta-analytic review. Personality and Individual Differences, 32(1), 15-25. Recuperado em 01 dezembro, 2018, de: https://doi.org/10.1016/S0191-8869(00)00233-6.

Saroglou, V., \& Muñoz-García, A. (2008). Individual Differences in Religion and Spirituality: An Issue of Personality Traits and/or Values. Journal for the Scientific Study of Religion, 47(1), 83-101. Recuperado em 01 dezembro, 2018, de: https://doi.org/10.1111/j.14685906.2008.00393.x.

Sattler, C., Toro, P., Schönknecht, P., \& Schröder, J. (2012). Cognitive activity, education and socioeconomic status as preventive factors for mild cognitive impairment and Alzheimer's disease. Psychiatry Research, 196(1), 90-95. Recuperado em 01 dezembro, 2018, de: https://doi.org/10.1016/j.psychres.2011.11.012. 
Smith, G. E., Petersen, R. C., Parisi, J. E., Ivnik, R. J., Kokmen, E., Tangalos, E. G., \& Waring, S. (1996). Definition, course, and outcome of mild cognitive impairment. Aging, Neuropsychology, and Cognition, 3(2), 141-147. Recuperado em 01 dezembro, 2018, de: https://doi.org/10.1080/13825589608256619.

Sobral, M., \& Paúl, C. (2015). Reserva Cognitiva, envelhecimento e demências. Revista E-Psi, 1(5), 113-134. Recuperado em 01 dezembro, 2018, de: https://revistaepsi.com/wpcontent/uploads/artigos/2015/Ano5-Volume1-Artigo6.pdf.

Souza, L. C., \& Teixeira, A. L. (2013). Envelhecimento patológico do sistema nervoso. In L. F. Malloy-Diniz, D. Fuentes, \& R. M. Cosenza (Orgs.). Neuropsicologia do Envelhecimento: Uma abordagem multidimensional (Cap. 5, 100-114). Porto Alegre, RS: Artmed.

Staff, R. T., Murray, A. D., Deary, I. J., \& Whalley, L. J. (2004). What provides cerebral reserve? Brain, 127(5), 1191-1199. Recuperado em 01 dezembro, 2018, de: https://doi.org/10.1093/brain/awh144.

Stern, Y. (2002). What is cognitive reserve? Theory and research application of the reserve concept. Journal of the International Neuropsychological Society, 8(3), 448-460. Recuperado em 01 dezembro, 2018, de: https://doi.org/10.1017/S1355617702813248.

Stern, Y., Arenaza-Urquijo, E. M., Bartrés-Faz, D., Belleville, S., Cantilon, M., Chetelat, G., ... Vuoksimaa, E. (2018). Whitepaper: Defining and investigating cognitive reserve, brain reserve, and brain maintenance. Alzheimer's and Dementia, 14(9), 1-7. Recuperado em 01 dezembro, 2018, de: https://doi.org/10.1016/j.jalz.2018.07.219.

Sutin, A. R. (2017). Openness. In: T. A. Widiger. (Ed.). The Oxford Handbook of the Five Factor Model (Cap. 5, 83-104). New York, USA: Oxford University Press.

Tupes, E. C., \& Christal, R. E. (1992). Recurrent Personality Factors Based on Trait Ratings. Journal of Personality, 60(2), 225-251. Recuperado em 01 dezembro, 2018, de: https://doi.org/10.1111/j.1467-6494.1992.tb00973.x.

Vasconcellos, S. J. L. (2007). Os pressupostos evolutivos dos fatores abertura à experiência e neuroticismo. Tese de doutorado, Universidade Federal do Rio Grande do Sul. Recuperado em 01 dezembro, 2018, de: https://www.lume.ufrgs.br/bitstream/handle/10183/ 14918/000672912.pdf? sequence $=1$.

Vasconcellos, S. J. L., \& Hutz, C. S. (2008). Construção e validação de uma escala de abertura à experiência. Avaliação Psicológica, 7(2), 135-141. Recuperado em 01 dezembro, 2018, de: http://pepsic.bvsalud.org/pdf/avp/v7n2/v7n2a04.pdf.

Recebido em 02/12/2019

Aceito em 30/12/2019 
Joel Jonathan Carvalho Tavares - Graduando em Psicologia, Centro Universitário de João Pessoa (UNIPÊ). Membro do Serviço de Neuropsicologia do Envelhecimento (SENE).

ORCID iD: https://orcid.org/0000-0001-9634-4884.

URL: https://www.unipe.edu.br

E-mail: joel@jonathan.net.br

Jayana Ramalho Ventura - Graduada em Psicologia, Centro Universitário de João Pessoa (UNIPÊ). Especialista em Psicologia Cognitivo-Comportamental, Centro Integrado de Tecnologia e Pesquisa (CINTEP/PB). Mestre em Neurociência Cognitiva e Comportamento, Universidade Federal da Paraíba (UFPB). Atualmente é Professora do Curso de Psicologia, Centro Universitário de João Pessoa (UNIPÊ).

URL: https://www.unipe.edu.br

E-mail: jayanarv@gmail.com

Bernardino Fernández-Calvo - Graduação e Licenciatura em Psicologia, Faculdade de Psicologia da Universidade de Salamanca (Espanha). Doutorado em Psicologia Clínica e da Saúde, Universidade de Salamanca. Atualmente é Professor Adjunto I no Departamento de Psicologia da Universidade Federal da Paraíba (UFPB) e Coordenador do Serviço de Neuropsicologia do Envelhecimento (SENE).

ORCID iD: https://orcid.org/0000-0001-8080-5578.

URL: https://sigaa.ufpb.br/sigaa/public/docente/portal.jsf?siape=2745539.

E-mail: bfcalvo@usal.es 